\title{
The nature of expertise in fingerprint examiners
}

\author{
Thomas A. Busey and Francisco J. Parada \\ Indiana University, Bloomington, Indiana
}

\begin{abstract}
Latent print examinations involve a complex set of psychological and cognitive processes. This article summarizes existing work that has addressed how training and experience creates changes in latent print examiners. Experience appears to improve overall accuracy, increase visual working memory, and lead to configural processing of upright fingerprints. Experts also demonstrate a narrower visual filter and, as a group, tend to show greater consistency when viewing ink prints. These findings address recent criticisms of latent print evidence, but many open questions still exist. Cognitive scientists are well positioned to conduct studies that will improve the training and practices of latent print examiners, and suggestions for becoming involved in fingerprint research are provided.
\end{abstract}

Latent print examiners face a daunting task. Fingerprints are often the only physical evidence left at a crime scene, but these are typically invisible until developed and lifted. Even after they are dusted with lifting powder and stabilized using clear lifting tape or fixed using cyanoacrylate fumes, friction ridge impressions may be corrupted by visual noise or may have missing regions. In some cases, it may even be difficult to orient the print correctly. This impression of the print must then be compared against a known standard, which can be either an ink print of a suspect developed by the investigating detective or one provided by a donor who has previously entered into the criminal justice system, whose prints now reside in a computerized database.

Although computers are used to store databases of fingerprints and to suggest possible matches, the amount of distortion and noise in latent prints prevents computer algorithms from performing near the level of human examiners. Thus, virtually all evidence admitted in courts comes from the testimony of experts. It is up to the examiners to decide whether they believe that there exists sufficient evidence to conclude that the two impressions (the latent and the ink print) come from the same source or that it can be excluded that they come from the same source.

The decision itself is fraught with complications. To conclude that the two impressions come from the same source implies that one has compared the latent print with all possible donors, which could be interpreted as all people who were in a position to leave the impression at the particular location. This set is potentially quite large, making any conclusion about individuality quite problematic. The flip side of this argument is that fingerprints are unique (as are all patterns in nature) and even identical twins have differentiable friction ridge impressions (Jain, Prabhakar, \& Pankanti, 2002).
Latent print examiners are caught between these two philosophical extremes, and given that skin may change through scars or wear, many practitioners now favor the term persistence over uniqueness. The field has had to contend with a steady stream of criticism, most recently from a National Research Council of the National Academy of Sciences (2009) report that highlighted what the panel saw as serious deficiencies in the practices of forensic scientists. However, even the most ardent critics of fingerprint identification would likely agree that there is some value in latent prints, and the aim of criticism is to address some of the shortcomings of current approaches.

Cognitive scientists have a role to play in this process, and the goal of this article is to discuss some of the major issues confronting the applied science of latent print examinations. We will review some of the nascent research on expertise among latent print examiners, as well as related research from cognitive psychology. This article focuses on the perceptual expertise side of latent print examinations; a companion article in this special issue by Dror and Cole (2010) addresses the cognitive biases that can affect the decision process, among other issues. The two articles focus on different aspects of latent print examinations, but both articles argue that the field itself has a large number of open questions and is in great need of assistance from cognitive scientists. One of our aims in writing this article is to illustrate how basic expertise research in the lab can impact the forensic sciences, and we will offer suggestions on how to become involved in research on these topics.

\section{OPEN QUESTIONS}

We organize this article around questions that have come from practitioners who are looking to improve their discipline, from critics who point out shortcomings from

T.A. Busey, busey@indiana.edu; F. J. Parada, fjparada@indiana.edu 
legal or logical standpoints, and from court decisions that have pushed for improvements in the practices of latent print examiners. In some cases, there is already a literature in which these questions are addressed, whereas in the majority of cases, the answers await empirical inquiry. Empirical approaches to these questions are relatively rare, although this is changing, and we will discuss contact points between the legal setting and the cognitive science community. Because some of the terminology may be unfamiliar to readers, we have defined some of the terms in the text box below.

\section{How Do Experts Conduct Latent Print Examinations?}

Individual laboratories may differ, but in general, an examiner will receive a set of latent prints from an investigating detective or a crime scene investigation (CSI) unit, along with a set of ink prints from a potential suspect. If no suspect is provided, the latent print may be entered into an automated database search engine, which uses the locations of minutiae and, sometimes, other sources of information to suggest a set of 10-20 candidate matches. The examiner will then spend time examining the latent print, often drawing or tracing the ridges. During this process, the examiner also determines which latent prints are deemed to be of value. The traditional approach is to look at the actual lift cards, using a magnifying glass, although many labs are going to digital images on LCD monitors.

Many examiners subscribe to an approach to the examination called ACE-V (Vanderkolk, 2009), which stands for analysis, comparison, evaluation, and verification. This methodology, which has been criticized as nothing more than an acronym (Zabell, 2005), provides some structure to the examination process. The examiner first studies the latent print and then looks for matching or mismatching detail in a candidate ink print. No two impressions will be identical, and the distortion caused by differences in pressure and skin contact requires that the examiner decide whether deviations in the locations of features are explainable by realistic physical processes, such as the ability of the skin to stretch without sliding. Some features are immune to distortions, such as counting the number of intervening ridges between two minutiae, and if an unexplainable difference is discovered, the examiner must exclude the suspect as the donor for the latent print.

European countries often have a numeric criterion for how many minutiae, or points, must match, whereas U.S. examiners are free to use whatever detail they deem of value. This implies that, at some level, the comparison process is a similarity judgment followed by a comparison with an internal criterion. Some examiners describe this process as one in which they ask whether these two prints are more similar than any other close nonmatch that they have encountered.

Experts typically testify to one of three conclusions: The two impressions come from the same donor (individualization), the two impressions did not come from the same donor (exclusion), or there was insufficient detail to determine a conclusion (inconclusive). The examiner may also issue a statement of confidence, some of which have been criticized as prejudicial (e.g., "100 percent confident," "zero percent error rate"; Cole, 2005).

\section{Text Box}

Core

Delta

Erroneous identification

Error rate

Forensic sciences

Friction ridge impressions Gabor patches

Holistic approach

Inked print

Latent print

Minutiae or point

Missed identification

Ridge line

Uniqueness
The portion of the middle of a fingerprint where the ridge begins to recurve back on itself.

A point of divergence of ridge lines to the left or right of the core, roughly triangular in appearance.

An error made by an examiner in which it is claimed that two impressions were made by the same individual, where, in fact, they were made by different individuals. These errors are typically discovered using an additional technology, such as DNA.

A term typically requested by courts with respect to a forensic discipline, but difficult to supply. This can take several forms, but the most common usage would be the number of erroneous identifications, relative to the total number of cases worked by an examiner.

A general term for a collection of applied sciences that use physical evidence in order to link a specific individual with a particular crime. This can include fingerprints and other skin impressions; firearm, tool, tire, bite, and cutting tool marks; footwear impressions; blood spatter analysis; and fracture matching for glass, tape, and plastic bags.

Impressions made by ridges found on fingers and toes that are thought to improve the friction for grasping objects.

Visual stimuli that are created by spatially cropping a sine wave grating with a Gaussian window. These have a very simple representation in the spatial frequency domain and, therefore, are likely processed by relatively few visual channels.

An approach to individualizations in the U.S. in which no fixed number of matching minutiae are required. The examiner tends to rely on their training and exposure to other fingerprints to determine the features that will lead to an individualization or exclusion.

A print typically made by law enforcement, using ink or a scanner. The source of the impression is typically known.

An impression typically recovered from a crime scene, using development procedures that highlight ridge detail. The source is typically unknown.

A region of the fingerprint where ridges either come together (bifurcation) or end (ridge ending). The location of minutiae can be diagnostic when comparisons are performed and can provide landmarks during the process.

An error made by an examiner in which two impressions come from the same source but the examiner claims that they did not.

A continuous set of ridge elements.

The philosophical supposition that no two physical objects can be identical. Within latent prints, uniqueness is limited by the quality of the impressions, no two of which can be identical, even from the same finger. 


\section{Are Experts Better Than Novices and, If So, How?}

The process described above has been described as overly subjective, since the evidence used to make a determination may vary from expert to expert. This raises the issue of whether experts can reliably match latent to ink prints at rates greater than novices and, if so, what psychological or physiological changes support this greater accuracy.

Relatively little literature exists on whether experts outperform novices in testing conditions that mimic latent print examinations. Recent work by Wertheim, Langenburg, and Moenssens (2006) demonstrated that experts with more than 1 year of training had an erroneous individualization (i.e., accusing an innocent person) rate of 0.034 percent out of all individualizations performed. However, the examiners also had what the researchers described as a clerical error rate of $1 \%$. The source of these errors is unclear, and the methods by which they were resolved were unconventional (including discussing the errors with the participants to determine the source of the error).

There is evidence that novices can match ink prints at above-chance levels (Vokey, Tangen, \& Cole, 2009) in abbreviated comparisons. Busey and Vanderkolk (2005) tested examiners with fragments of fingerprints that could be partially masked and/or corrupted by visual noise. The examiners were shown a target fingerprint for $1 \mathrm{sec}$ and then, after a delay of $200 \mathrm{msec}$ or $5 \mathrm{sec}$, were shown two new fingerprints that included a matching print and a distractor print. We used this task to focus on the perceptual aspects of expertise, rather than decisional factors. The distractor prints were carefully chosen to appear quite similar to the target, and features such as scars or creases were eliminated to encourage the participants to rely on ridge structure. The experts performed reliably better than the novices at identifying the matching print and were almost unaffected by the 5-sec delay. In addition, the experts performed much better on the full fragments in noise than on the partial fragments in noise, and multinomial processing tree analyses demonstrated that this improvement was above what would be predicted by probability summation models. We interpreted this result as evidence for configural processing, in which the information in one half of the print becomes more useful in the presence of the second half.

This result was confirmed in a second study using electrophysiological recording. The N170 ERP component is reliably delayed and enhanced when stimuli that are thought to be processed using configural processing are inverted. Faces (Eimer, 2000) and trained stimuli such as Greebles (Rossion et al., 2000) are examples of these stimuli. Examiners almost always view fingerprints in an upright orientation and, therefore, may develop holistic representations only at this orientation. We inverted the fingerprints during categorization and identification tasks and found that it produced a reliable delay in the N170 ERP component, suggesting configural processing of upright fingerprints (Busey \& Vanderkolk, 2005). This opens up the possibility that experts may encode configurations of features, rather than simply matching individual minutiae. Work by Schyns and Rodet (1997) has suggested that ex- perience can alter the featural representation of a learned stimulus set, and Goldstone (2000) argued that unitization can create new features. Thus, one aspect of expertise that would support greater accuracy may be the development of new feature sets or configural mechanisms.

Empirical data collected as part of abbreviated 20-sec latent print examinations where participants determined whether two prints matched (Busey et al., 2010) showed a mean $d^{\prime}$ for experts of 2.47 and a mean $d^{\prime}$ for novices of 1.30. Thus, experts seem to be able to reliably outperform novices and have fairly high $d^{\prime}$ values even with short viewing times.

Despite all this evidence, no overall error rate for latent print identification is currently known and would probably be lab- and examiner-dependent. Although examiners undergo proficiency testing in some labs, it is difficult to calibrate the difficulty of these exams against that required by casework, and laboratories may give more difficult cases to more practiced examiners. All of these factors work against a single summary of the overall quality of fingerprint evidence, which is not to say that we cannot work toward an understanding of how pervasive errors are and how to reduce them. Indeed, the National Institute of Standards and Technology and the National Institute of Justice have jointly commissioned a working group to identify the human factors that affect accuracy in latent print examinations.

\section{What Features or Sources of Information Do Experts Use?}

One approach to the question of what information experts use is to rely on eyetracking procedures (Busey et al., 2010). In the medical imaging discipline, this technique has proven useful for determining the features that are used to diagnose breast cancer (Krupinski, 1996). We tested experts using a portable eyetracker in abbreviated 20 -sec trials, during which they searched for the most diagnostic features in order to decide whether two prints came from the same source. We addressed the question of whether experts as a group show more consistency than do novices. Somewhat surprisingly, we found that with latent ink comparisons (where one print is taken from the field and one from an arrest record), experts show more variability than do novices. Novices may all look for the relatively small regions of the latent prints that are fairly clear, whereas experts may venture off into less clear regions in order to identify diagnostic features. We repeated this test with ink prints that were free of visual noise and found the opposite result: Experts as a group were now more consistent, despite the fact that the ink prints presented a wealth of information to choose from. Part of the reason for this consistency comes from the fact that the experts tended to focus on the core and delta regions of the fingerprint.

Figure 1 illustrates an example pair of prints in which experts show greater consistency than do novices. These results suggest that, among examiners, there is an implicit understanding of which regions are the most diagnostic and they visit these first. However, if the information is constrained by external noise, different examiners will examine different regions to identify detail that best suits their style. 

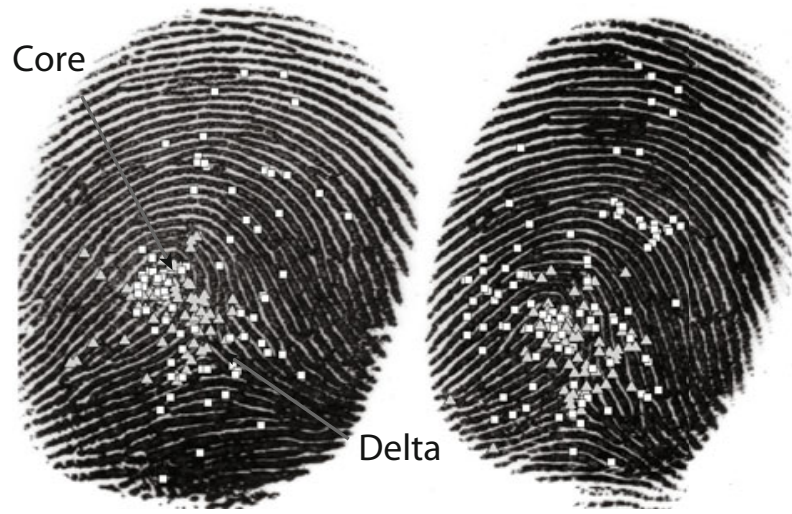

Figure 1. Eye fixations for six experts (gray triangles) and seven novices (white squares) from one matching image pair. Note that the expert data tends to cluster near the core and delta, whereas the novice data are much more variable.

Experts appear to make smaller saccades when viewing latent and ink prints, which suggests that they are accumulating information across regions or following individual ridges. More sophisticated analyses of the actual image information will reveal the feature set that experts rely on, which requires a choice of data reduction algorithms. We are currently exploring several candidate techniques. One approach relies on feature induction procedures, which have been successful in more restricted domains (Cohen, Shiffrin, Gold, Ross, \& Ross, 2007).

We have addressed the question of what sources of information experts rely on, using noise-masking procedures (Busey, Schneider, \& Wyatte, 2008). We used bandpass filtered noise in a forced choice identification task in which the filtered noise was added to the study stimulus and then the observer chose from two clear test prints. The logic of this design is that the noise will knock out a specific range of spatial frequencies and, if performance suffers when the noise is added to the image, we can infer that participants were using information in that spatial frequency band. Fingerprints have a characteristic bump in the amplitude spectrum that corresponds to the typical width of the ridges. We tested experts and novices, using faces and fingerprints, and recorded electrophysiological data during the presentation of the stimulus embedded in noise. The range of noise that affects performance gives an estimate of the width of the visual filter, since it describes the range of spatial frequencies that allowed good performance when seen without a mask. We found that fingerprint examiners had narrower visual filters than did novices, but only for fingerprints. The narrower visual filter was found both for behavioral accuracy and for the EEG activity recorded over the right parietal region at about $250 \mathrm{msec}$ after stimulus onset. We interpreted these results in terms of experts' learning to ignore spatial frequency channels that had low signal-to-noise ratios and, therefore, being unaffected by noise added in these shoulder channels next to the highly informative channels for fingerprints.

\section{How Much Evidence Is Required?}

Once examiners select a feature set, they must decide how much evidence is required before a match or exclusion can be determined. Friction ridge impressions can be viewed as containing information at multiple scales. The field has acknowledged this by labeling the overall ridge flow as Level 1 detail; individual ridge paths, path lengths, and the locations of features such as ridge endings and bifurcations as Level 2 detail; and the shape or textures of individual ridge elements as Level 3 detail. Examiners' reports typically rely on Level 2 detail, but there is a published case study of an individualization of a fingerprint fragment despite the lack of any minutiae or ridge endings (Reneau, 2003). Current approaches allow the examiner to determine the level of sufficiency that they require, along with the feature set that they rely on.

The limitation of this approach is that experts may rely on features that are common to large numbers of impressions and, therefore, do not appropriately individualize any one print. In addition, the amount of evidence required to make a determination may vary from expert to expert. Critics have argued that this is needlessly subjective (Epstein, 2002), and the National Research Council of the National Academy of Sciences (2009) report called for additional research to quantify the nature of information available in latent prints.

Statistical modeling approaches to information quantification. To address these concerns, several authors have developed statistical models to either demonstrate the likelihood of an erroneous identification or document the diagnosticity of particular features. These approaches typically rely on easily measured features, such as minutiae locations and orientations or path lengths.

Neumann et al. (2007) developed a statistical model in which the locations and orientations of minutiae are perturbed using a distortion model to create new instances of an existing print, analogous to capturing a range of latent prints under different conditions. The similarity between a known set of prints and a new print is measured, and the model determines the likelihood that the most similar print in the database comes from the donor of the new print. Thus, the model essentially performs the identification process (albeit with high-quality prints), and its accuracy can be measured. The results indicate that the likelihood of an erroneous identification with high-quality ridge information becomes vanishingly small, although this depends on the population one is sampling from and the rarity of the features in the latent print.

The conclusions derived from this approach depend heavily on the distortion model applied to individual impressions. If large distortions are allowed, the strength of evidence is weakened, and the chance of a false identification is greater. The authors based their distortion model, in part, on empirical tests of skin stretching, but further testing will be required before field deployment is warranted.

A second approach is to measure the population parameters of different minutiae. Su and Srihari (2008) measured the locations and orientations of minutiae and ridge 
elements to develop a generative model for fingerprints. A small amount of error or distortion is allowed around each minutia, and new impressions that are not yet in the database can be normalized and compared against all prints in the database to determine how common or rare minutiae are in each location. For example, on left or right loop patterns, minutiae on the side opposite of the delta tend to give very similar patterns across fingerprints and, therefore, may not be as diagnostic as those in other regions, such as the core.

Both of these approaches demonstrate that the information content of good quality fingerprints is quite high. Recently, a group at IBM demonstrated that a fingerprint with 25 minutiae is equivalent to a 16-character nonsense password, or about 82 bits of information (Ratha, Connell, \& Bolle, 2001). Thus, without significant noise or distortions, fingerprints theoretically contain a great deal of identifying information.

Despite these quantitative analyses, sufficiency for identification remains an individual decision, based on what the examiners typically describe as their "training and experience with matching and nonmatching prints." There currently exists no statement of sufficiency in the field, and professional organizations such as the Scientific Working Group for Friction Ridge Analysis, Study, and Technology have stated that "there is no scientific basis for requiring that a predetermined number of corresponding friction ridge details be present in two impressions in order to effect individualization" (SWGFAST, 2003, Standards for Conclusions, section 1.2.1).

\section{What Methods Are Best for \\ Training New Examiners?}

In addition to learning which features or dimensions to attend to, examiners may undergo alterations in the perceptual template that weights different perceptual channels. Dosher and Lu (2005) addressed the role of different perceptual-learning mechanisms, using Gabor patches that were similar in appearance to latent print fragments. They asked participants to perform an orientation discrimination task and found a contrast threshold for both low-noise and high-noise conditions. Critically, some participants were trained first in low-noise conditions and then tested in high-noise trials, whereas others were run in the reverse order. Training in low-noise conditions generalized to high noise, whereas the reverse was not true. Dosher and Lu interpreted this in terms of separable mechanisms: Learning in low noise both improves the perceptual representation of the stimulus and helps exclude external noise, whereas learning in high noise affects only the external noise exclusion mechanism. Thus, training should initially involve only clear prints that are not corrupted by visual noise, which allows the visual system to interpret the set of available features.

\section{BECOMING INVOLVED IN RESEARCH ON LATENT PRINTS}

We have found that fingerprint examinations involve a complex set of perceptual and cognitive capacities, each of which is potentially altered by expertise. As the studies above demonstrate, it is possible to apply the tools of cognitive science to specific questions related to these capacities. Fingerprints are remarkably similar to faces, in that they are defined by a limited set of features that tend to occur in characteristic locations. Although fingerprints are not as alignable as faces, experts exhibit similar mechanisms, such as configural processing, when perceiving them. In addition, they have the advantage that the evidence is physical in nature, unlike eyewitness testimony, in which the evidence is locked inside the memory of the eyewitness. Thus, those who have worked with faces may find latent prints to be familiar territory.

Research on forensic identification need not be limited to fingerprints. Recently, Vanderkolk (2009) argued that all patterns in nature are unique and that individualizations are limited only by the quality of the impression left behind. He argued that lip prints, cheek prints, and even glass fractures and paper tears can be individualized by an examiner who takes the time to understand what is unique about the patterns.

Research on latent print examinations also provides the opportunity to extend basic science results outside the laboratory and apply them within an ongoing legal debate. It is our experience that fingerprint examiners are guardedly open to collaborating with researchers. They are extremely sensitive to the criticism that has been leveled at their field, but a growing consensus seems to be emerging that there is room for improvement in both training and practices.

To have the most impact, care should be taken when experiments are designed. Perhaps most critical is the choice of distractors and the base rate of nonmatching prints. The increasing use of computerized databases has produced candidate ink prints that can look remarkably similar to a latent print even if they come from different donors. In addition, the error rate of examiners is, in large part, determined not by the easy exclusions based on obviously different classes of patterns, but by the difficult comparisons where the overall ridge structure is similar. These experimental designs will allow us to address questions that are most important to the field, such as what causes errors, how reliable is the evidence, and what conclusions can be drawn.

\section{AUTHOR NOTE}

This research was supported by NIJ Grants 2005-MU-BX-K076 and 2009-DN-BX-K226 to the first author. Correspondence concerning this article should be addressed to T. A. Busey or F. J. Parada, Department of Psychology, Indiana University, Bloomington, IN 47405 (e-mail: busey@indiana.edu or fjparada@indiana.edu).

\section{REFERENCES}

Busey, T. [A.], Schneider, B., \& Wyatte, D. (2008, May). Expertise and the width of the visual filter in fingerprint examiners. Poster presented at the 8th Annual Meeting of the Vision Sciences Society, Naples, FL.

BusEy, T. A., \& VANDERKOLK, J. R. (2005). Behavioral and electrophysiological evidence for configural processing in fingerprint experts. Vision Research, 45, 431-448.

Busey, T. A., Yu, C., Wyatte, D., Vanderkolk, J., Parada, F. J., \& Akvipat, R. (2010). Consistency and variability among latent print examiners as revealed by eye tracking methodologies. Manuscript submitted for publication. 
Cohen, A. L., Shiffrin, R. M., Gold, J. M., Ross, D. A., \& Ross, M. G. (2007). Inducing features from visual noise. Journal of Vision, 7(8, Art. 15), 1-14

Cole, S. A. (2005). More than zero: Accounting for error in latent fingerprint identification. Journal of Criminal Law \& Criminology, 95, 985-1078.

Dosher, B. A., \& Lu, Z.-L. (2005). Perceptual learning in clear displays optimizes perceptual expertise: Learning the limiting process. Proceedings of the National Academy of Sciences, 102, 5286-5290.

Dror, I. E., \& Cole, S. A. (2010). The vision in "blind" justice: Expert perception, judgment, and visual cognition in forensic pattern recognition. Psychonomic Bulletin \& Review, 17, 161-167.

EIMER, M. (2000). Effects of face inversion on the structural encoding and recognition of faces: Evidence from event-related brain potentials. Cognitive Brain Research, 10, 145-158.

Epstein, R. (2002). Fingerprints meet Daubert: The myth of fingerprint "science" is revealed. Southern California Law Review, 75, 605-657.

Goldstone, R. L. (2000). Unitization during category learning. Journal of Experimental Psychology: Human Perception \& Performance, 26, $86-112$.

Jain, A. K., Prabhakar, S., \& Pankanti, S. (2002). On the similarity of identical twin fingerprints. Pattern Recognition, 35, 2653-2663.

KRUPINSKI, E. A. (1996). Visual scanning patterns of radiologists searching mammograms. Academic Radiology, 3, 137-144.

National Research Council of the National Academy of ScIENCES (2009). Strengthening forensic science in the United States: A path forward. Washington, DC: National Academy of Sciences.

Neumann, C., Champod, C., Puch-Solis, R., Egli, N., Anthonioz, A., \& Bromage-Griffiths, A. (2007). Computation of likelihood ratios in fingerprint identification for configurations of any number of minutiae. Journal of Forensic Sciences, 52, 54-64.

Ratha, N. K., Connell, J. H., \& Bolle, R. M. (2001). An analysis of minutiae matching strength. In J. Bigun \& F. Smeraldi (Eds.), Audioand video-based biometric person authentication (Lecture Notes in Computer Science, Vol. 2091, pp. 223-228). Berlin: Springer.

RENEAU, R. D. (2003). Unusual latent print examinations. Journal of Forensic Identification, 53, 531-537.

Rossion, B., Gauthier, I., Tarr, M. J., Despland, P., Bruyer, R., LiNOTTE, S., \& CrommelincK, M. (2000). The N170 occipito-temporal component is delayed and enhanced to inverted faces but not to inverted objects: An electrophysiological account of face-specific processes in the human brain. NeuroReport, 11, 69-74.

SchYNs, P. G., \& RodET, L. (1997). Categorization creates functional features. Journal of Experimental Psychology: Learning, Memory, \& Cognition, 23, 681-696.

Su, C., \& SRIHARI, S. N. (2008, December). Generative models for fingerprint individuality using ridge models. Paper presented at the International Conference on Pattern Recognition, Tampa, FL.

SWGFAST (2003). Standards for conclusions. Available at www.swgfast .org.

VANDERKolK, J. R. (2009). Forensic comparative science: Qualitative, quantitative source determination of unique impressions, images, and objects. Burlington, MA: Elsevier.

Vokey, J. R., TANGen, J. M., \& Cole, S. A. (2009). On the preliminary psychophysics of fingerprint identification. Quarterly Journal of Experimental Psychology, 62, 1023-1040.

Wertheim, K., Langenburg, G., \& Moenssens, A. (2006). Report of latent print examiner accuracy during comparison training exercises. Journal of Forensic Identification, 56, 55-127.

Zabell, S. (2005). Fingerprint evidence. Journal of Law \& Policy, 13, 143-179.

(Manuscript received June 26, 2009; revision accepted for publication October 17, 2009.) 